\title{
Electromagnetic Modeling of Split-Ring Resonators
}

\author{
Levent Gürel $^{1,2}$, Alper Ünal ${ }^{1}$, and Özgür Ergül ${ }^{1}$ \\ ${ }^{1}$ Department of Electrical and Electronics Engineering \\ ${ }^{2}$ Computational Electromagnetics Research Center (BiLCEM) \\ Bilkent University, TR-06800, Bilkent, Ankara, Turkey \\ E-mail: lgurel@bilkent.edu.tr, runal@ug.bilkent.edu.tr,ergul@ee.bilkent.edu.tr
}

\begin{abstract}
In this paper, we report our efforts to model splitring resonators (SRRs) and their large arrays accurately and efficiently in a sophisticated simulation environment based on recent advances in the computational electromagnetics. The resulting linear system obtained from the simultaneous discretization of the geometry and Maxwell's equations is solved iteratively with the multilevel fast multipole algorithm. As an example, we present an array of 125 SRRs showing a negative effective permeability about $92 \mathrm{GHz}$.

Index Terms - Metamaterials, split-ring resonators, multilevel fast multipole algorithm.
\end{abstract}

\section{INTRODUCTION}

Since they were first proposed theoretically by Veselago in 1968 [1], metamaterials (MMs) have attracted a great amount of interest because of their unusual electromagnetic properties. MMs are constructed by embedding periodic inclusions, such as split-ring resonators (SRRs), into a host medium. In this paper, we report our efforts to simulate SRR structures accurately and efficiently. In our modeling and simulations, we take into account that these structures actually have finite extent and they exhibit interface properties. Without using symmetry and homogenization, we accurately model large number of inclusions to understand the scattering and transmission properties of these structures. Accurate modeling and fast solution of these three-

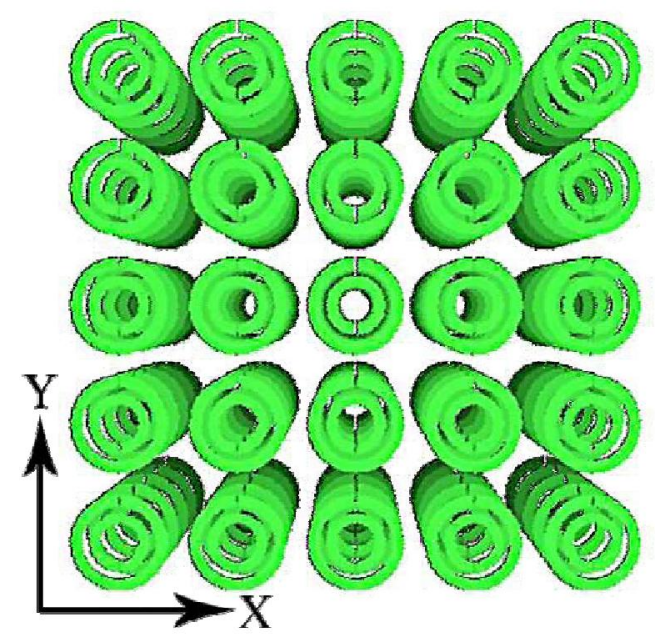

Fig. 1. Array of $5 \times 5 \times 5$ SRRs, which is expected to generate negative effective permeability into the medium around the resonance frequency. dimensional structures translate into very large computational problems, which can be solved with the aid of advanced acceleration techniques such as the multilevel fast multipole algorithm (MLFMA) [2].

\section{ELECTROMAGNETIC MODELING}

In this paper, we consider an example of $5 \times 5 \times 5$ SRR array depicted in Fig. 1, which is designed to have a resonance frequency about $100 \mathrm{GHz}$ [3]. Around the resonance frequency, the transmission through the array is expected to decrease significantly due to the negative effective permeability stimulated in the medium. Dimensions of a single SRR is as follows (Fig. 2): The smaller ring has $43 \mu \mathrm{m}$ inner radius and $67.2 \mu \mathrm{m}$ outer radius, the larger ring has 80.7 $\mu \mathrm{m}$ inner radius and $107.5 \mu \mathrm{m}$ outer radius, and the gap width is $7 \mu \mathrm{m}$. The SRR array in Fig. 1 is obtained by the arrangement of 125 SRRs with periodicities of $262.7 \mu \mathrm{m}$ in the $x$ and $y$ directions, and $150 \mu \mathrm{m}$ in the $z$ direction.

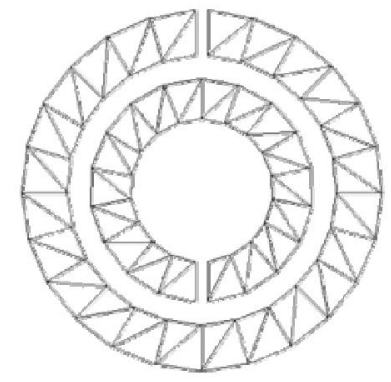

(a)

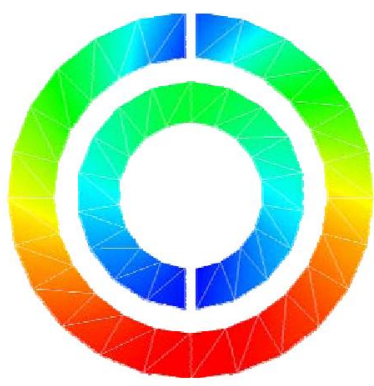

(b)
Fig. 2. Single SRR used to construct the array in Fig. 1.

(a) Triangulation applied for optimal accuracy and efficiency.

(b) Current density induced on a single SRR at $100 \mathrm{GHz}$. (Red and blue represent high and low values, respectively.)

In the simulation environment, the electric-field integral equation (EFIE) is employed to formulate the electromagnetic problem. For conducting surfaces, the boundary condition for the tangential electric field can be used to derive EFIE as

$$
\hat{t} \cdot \int_{S} d \boldsymbol{r}^{\prime} \boldsymbol{J}\left(\boldsymbol{r}^{\prime}\right) \cdot\left(\overline{\boldsymbol{I}}-\frac{\nabla \nabla^{\prime}}{k^{2}}\right) g\left(\boldsymbol{r}, \boldsymbol{r}^{\prime}\right)=\frac{i}{k \eta} \hat{t} \cdot E^{i}(\boldsymbol{r}),
$$


where we assume the $\exp (-i w t)$ convention in phasor notation. In (1), scattered field is expressed in terms of the induced (unknown) current $\boldsymbol{J}\left(\boldsymbol{r}^{\prime}\right), \boldsymbol{r}$ is an observation point on the surface of the SRRs, $\hat{t}$ is any tangential vector to the surface at the observation point, $E^{i}(r)$ is the incident field, and

$$
g\left(\boldsymbol{r}, \boldsymbol{r}^{\prime}\right)=\frac{\exp \left(i k\left|\boldsymbol{r}-\boldsymbol{r}^{\prime}\right|\right)}{4 \pi\left|\boldsymbol{r}-\boldsymbol{r}^{\prime}\right|}
$$

denotes the free-space Green's function. As depicted in Fig. 2(a), SRRs are modeled by small triangles, on which RaoWilton-Glisson [4] basis functions are defined to expand the unknown current density induced on the metallic surfaces. To obtain the triangulation in Fig. 2(a), we performed convergence analysis for the optimal discretization in terms of the accuracy and efficiency, leading to 84 and 10,500 triangular elements for a single SRR and the entire array in Fig. 1, respectively.

By the simultaneous discretization of the geometry and EFIE, we obtain a dense matrix equation

$$
\sum_{n=1}^{N} Z_{m n} a_{n}=v_{m}, \quad m=1, \ldots, N,
$$

where the matrix elements are derived as

The matrix equation in (3) is solved iteratively, where the matrix-vector products are accelerated by the MLFMA and

$$
Z_{m n}=\int_{S_{m}} d \boldsymbol{r} \boldsymbol{t}_{m}(\boldsymbol{r}) \cdot \int_{S n} d \boldsymbol{r}^{\prime}\left(\overline{\boldsymbol{I}}-\frac{\nabla \nabla^{\prime}}{k^{2}}\right) g\left(\boldsymbol{r}, \boldsymbol{r}^{\prime}\right) \cdot \boldsymbol{b}_{n}(\boldsymbol{r})
$$

the number of iterations is reduced by a near-field preconditioner. Solving for the coefficients $a_{n}$, we obtain accurate representations for the induced surface currents as depicted in Fig 2(b) for a single SRR. In this paper, the SRR array is illuminated by a Hertzian dipole, although we also employ other excitations such as beam-like waves based on theoretical complex source point. The relative permittivity of the host medium is 4.8 , as it is commonly used in experimental setups [3].

\section{NUMERICAL RESULTS}

In Fig. 3, the transmission through the SRR array is plotted with respect to the frequency in the range from $85 \mathrm{GHz}$ to 110 GHz. Around $92 \mathrm{GHz}$, the transmitted power is reduced significantly due to the resonance of the SRR structure. This sharp change in the electromagnetic properties of the array is also confirmed in Fig. 4, where the total power distribution on the $z=0$ plane is presented for some of the frequencies. The transmission region is on the left of the SRR array as indicated in the plots. About $92 \mathrm{GHz}$, the total power in the transmission region drops dramatically due to the shadowing effect of the SRR structure.

\section{CONCLUSIONS}

This paper reports accurate and efficient modeling of large SRR arrays in a sophisticated simulation environment. As an example, we present a $5 \times 5 \times 5$ SRR array having a resonance around $92 \mathrm{GHz}$ thus creating negative effective permeability in the medium. Our solution techniques based on iterative methods accelerated with the MLFMA provide efficient and accurate modeling of much larger problems of SRR arrays and other components of MMs.

\section{ACKNOWLEDGEMENT}

This work was supported by the Turkish Academy of Sciences in the framework of the Young Scientist Award Program (LG/TUBA-GEBIP/2002-1-12), by the Scientific and Technical Research Council of Turkey (TUBITAK) under Research Grant 105E172, and by contracts from ASELSAN and SSM.

\section{REFERENCES}

[1] V. G. Veselago, "The electrodynamics of substances with simultaneously negative values of permittivity and permeability," Sov. Phys. Usp., vol 10, no. 4, pp. 509-514, Jan.-Feb. 1968.

[2] W. C. Chew, J.-M. Jin, E. Michielssen, and J. Song, "Fast and Efficient Algorithms in Computational Electromagnetics." Boston, MA: Artech House, 2001.

[3] M. Gokkavas, K. Guven, I. Bulu, K. Aydın, R. S. Penciu, M. Kafesaki, C. M. Soukoulis and E. Ozbay, "Experimental demonstration of a left-handed metamaterial operating at $100 \mathrm{GHz}$," Phys. Rev. B., vol 73, no. 1931032006.

[4] S. M. Rao, D. R. Wilton, and A. W. Glisson, "Electromagnetic scattering by surfaces of arbitrary shape," IEEE Trans. Antennas Propagat., vol. AP-30, no. 3, pp. 409-418, May 1982.

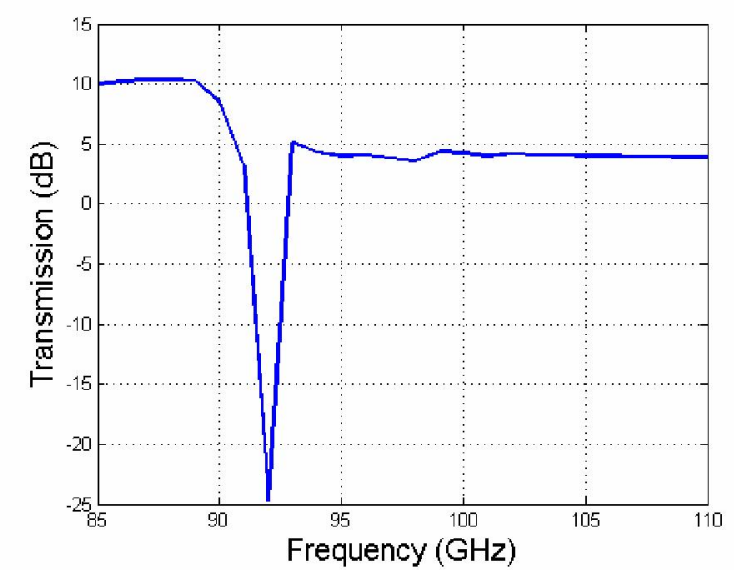

Fig. 3. Transmission through the SRR array in Fig. 1 with respect to frequency. 

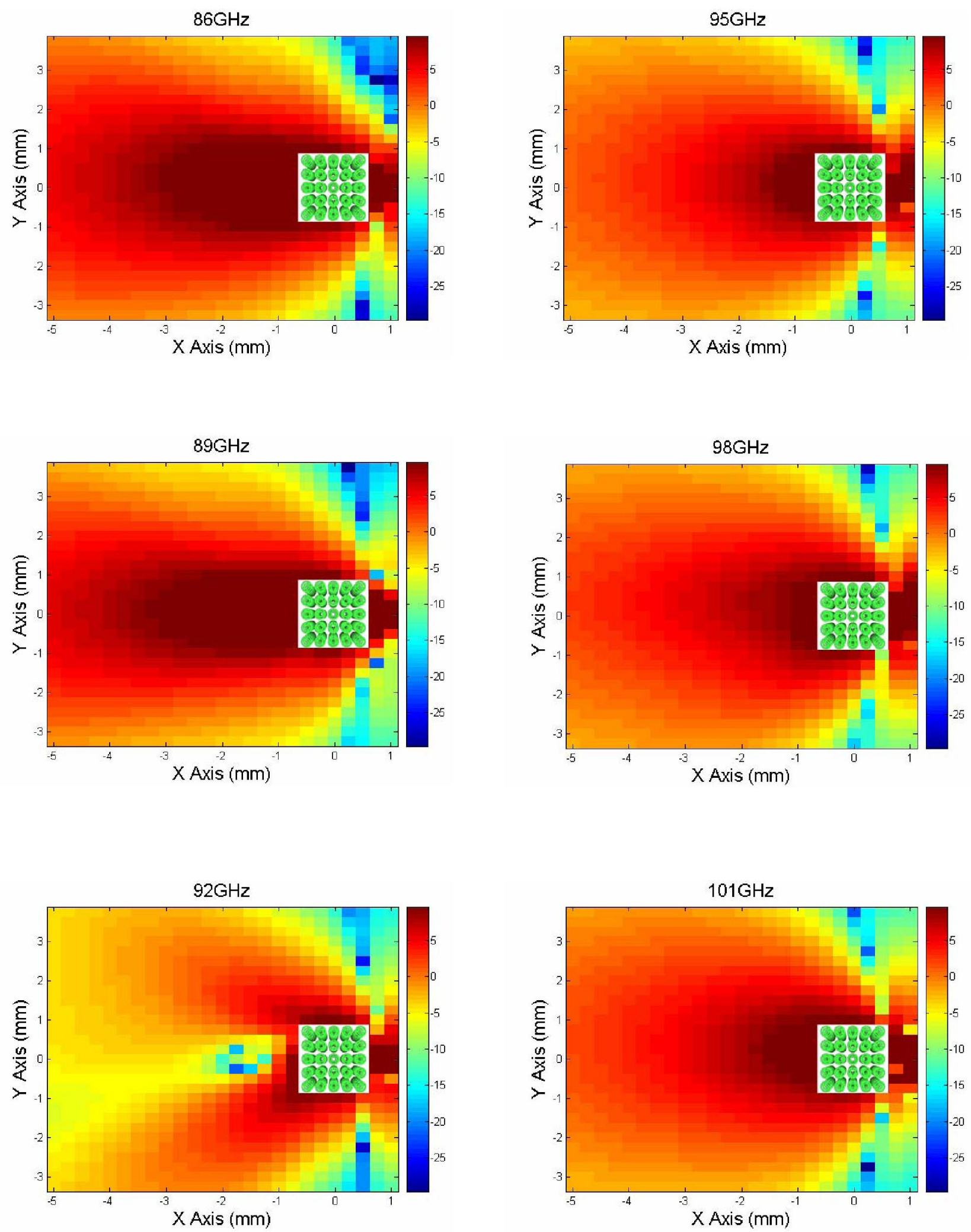

Fig. 4. Total power (dB) distribution for the SRR array in Fig. 1 illuminated by a Hertzian dipole located to the right hand side of the array. At $92 \mathrm{GHz}$, there exists a shadowing effect to the left hand side of the array. 